\title{
On some systems of conics connected with the triangle.
}

\author{
By Professor R. E. Allardice.
}

I have recently shown* that any two straight lines that are isotomically conjugate with reference to a triangle are the asymptotes of a conic circumscribed about the triangle; and that, conversely, any circumscribed conic has for its asymptotes two such lines. Now there is a one-fold infinity of conics having a given pair of straight lines for asymptotes; and, as there is a two-fold infinity of pairs of isotomic lines in the plane (with reference to a given triangle), it follows that two conditions must subsist among the coeflicients in the equation of a conic, in order that its asymptotes may be isotomic lines. In order to find these conditions, we may write the equation of the conic in the form

$$
\begin{aligned}
& (p a+q \beta+r \gamma)\left(a^{2} a / p+b^{2} \beta / q+c^{2} \gamma / r\right)+k(a \alpha+b \beta+c \gamma)^{2}=0, \\
& \text { or } \quad(k+1)\left(a^{2} a^{2}+b^{2} \beta^{2}+c^{2} \gamma^{2}\right)+\left(a^{2} q / p+b^{2} p / q+2 k a b\right) \alpha \beta+\ldots=0 ;
\end{aligned}
$$

and hence the conditions that the conic

$$
\mathrm{A} \alpha^{2}+\mathrm{B} \beta^{2}+\mathrm{C} \gamma^{2}+2 \mathrm{~F} \beta \gamma+2 \mathrm{G} \gamma \alpha+2 \mathrm{H} a \beta=0
$$

have a pair of isotomic lines for asymptotes (or, be similar, similarly situated and concentric with some circumscribed conic) are

$$
\mathrm{A}: \mathrm{B}: \mathrm{C}=a^{2}: b^{2}: c^{2}
$$

The equation of the system of conics that have the same asymptotes as the circumscribed conic $F \beta \gamma+G \gamma a+H \alpha \beta=0$, may be written

$$
\lambda(a a+b \beta+c \gamma)^{2}+\mathrm{F} \beta \gamma+\mathrm{G} \gamma a+\mathrm{H} a \beta=0 .
$$

It is obvious that any one of these conics meets each side of the triangle in points that are isotomically conjugate. If $\xi, \eta, \zeta$ are the intercepts made on the sides of the triangle by any conic of this system, we may show that

$$
1-(\xi / a)^{2}: 1-(\eta / b)^{2}: 1-(\zeta / c)^{2}=1 / \mathrm{F}: 1 / \mathrm{G}: 1 / \mathrm{H}=\text { constant. }
$$

* Annals of Mathematics, Second Series, Vol. 2, No. 3, p. 148 (April 1901). 
Writing down the condition that a conic of the above system be inscribed in the triangle, we find $\mathbf{F}=-4 \lambda b c$ or 0 , etc.; hence the only non-degenerate inscribed and circumscribed conics that are similar, similarly situated and concentric are the maximum inscribed ellipse $\quad a^{2} \alpha^{2}+b^{2} \beta^{2}+c^{2} \gamma^{2}-2 a b a \beta-2 b c \beta \gamma-2 c a \gamma a=0$, and the maximum circumscribed ellipse $b c \beta \gamma+c a \gamma a+a b a \beta=0$.

A similar investigation may be made to find the conditions that must subsist, in order that a conic shall be confocal with some inscribed conic, or, shall have a pair of isogonal points as foci. It is obvious, from the properties of confocal conics, that the tangents to the conic, drawn from the vertices of the triangle, must be isogonal conjugates.

Let the conic be

$$
\mathrm{A} a^{2}+\mathrm{B} \beta^{2}+\mathrm{C} \gamma^{2}+2 \mathrm{~F} \beta \gamma+2 \mathrm{G} \gamma a+2 \mathrm{H} a \beta=0 \text {, and put } a=\lambda \beta ;
$$

then

$$
\beta^{2}\left(\lambda^{2} \mathbf{A}+2 \lambda \mathbf{H}+\mathbf{B}\right)+2 \beta \gamma(\mathbf{F}+\lambda \mathbf{G})+\mathbf{C} \gamma^{2}=0 ;
$$

and the condition for equal roots is

$$
\left(\mathrm{G}^{2}-\mathrm{AC}\right) \lambda^{2}+2(\mathrm{FG}-\mathrm{CH}) \lambda+\left(\mathrm{F}^{2}-\mathrm{BC}\right)=0 .
$$

This equation determines the two values of $\lambda$ for which $a=\lambda \beta$ is a tangent; and in order that the two tangents thus obtained may be isogonal lines, we must have $G^{2}-A C=F^{2}-B C$. Hence the required conditions are

$$
\mathrm{F}^{2}-\mathrm{BC}=\mathrm{G}^{2}-\mathrm{CA}=\mathrm{H}^{2}-\mathrm{AB} .
$$

In finding the equation of the system of conics confocal with a given inscribed conic, it will be convenient to employ tangential coordinates, and to make use of the condition that the required system of conics must be concentric with the given conic.

Let the given inscribed conic be $\mathrm{F} \beta \gamma+\mathrm{G} \gamma \alpha+\mathrm{H} \alpha \beta=0$; any conic that has a pair of isogonal points as foci may be written

$$
\alpha^{2}+\beta^{2}+\gamma^{2}+2 \mathrm{~F}_{1} \beta \gamma+2 \mathrm{G}_{1} \gamma \alpha+2 \mathrm{H}_{1} \alpha \beta=0 \text {. }
$$

The equation of the centre of the latter conic, the pole of the straight line at infinity, $(a, b, c)$, is

$$
\left(a+\mathrm{G}_{1} c+\mathrm{H}_{1} b\right) a+\left(b+\mathrm{H}_{1} a+\mathrm{F}_{1} c\right) \beta+\left(c+\mathrm{F}_{1} b+\mathrm{G}_{1} a\right) \gamma=0 ;
$$

and the equation of the centre of the former conic is

$$
(\mathrm{G} c+\mathrm{H} b) a+(\mathrm{H} a+\dot{\mathrm{F}} c) \beta+(\mathrm{F} b+\mathrm{G} a) \gamma=0 .
$$


Hence

$$
\begin{aligned}
& a+\mathrm{G}_{1} c+\mathrm{H}_{1} b=\lambda(\mathrm{G} c+\mathrm{H} b), \\
& b+\mathrm{H}_{1} a+\mathrm{F}_{1} c=\lambda(\mathrm{H} a+\mathrm{F} c), \\
& c+\mathrm{F}_{1} b+\mathrm{G}_{1} a=\lambda(\mathrm{F} b+\mathrm{G} a) ;
\end{aligned}
$$

which give $F_{1}=\lambda F-\cos A, G_{1}=\lambda G-\cos B, H_{1}=\lambda H-\cos C$.

Thus the required equation is

$\alpha^{2}+\beta^{2}+\gamma^{2}-2 \beta \gamma \cos A-2 \gamma \alpha \cos B-2 \alpha \beta \cos C+2 \lambda(F \beta \gamma+G \gamma \alpha+H a \beta)=0$.

This result is otherwise obvious; for the part independent of $\lambda$, when equated to zero, gives the tangential equation of the circular points at infinity; and the above equation is equivalent to the statement that the conic it represents and the conic

$$
\mathrm{F} \beta \gamma+\mathrm{G} \gamma a+\mathrm{H} \alpha \beta=0
$$

are inscribed in the same quadrilateral, whose sides pass through the circular points at infinity.

If we transform the above equation from tangential to pointcoordinates, we get

$$
\begin{gathered}
{[\alpha \sin \mathrm{A}+\beta \sin \mathrm{B}+\gamma \sin \mathrm{C}]^{2}+2 \lambda\left[\mathrm{F} \cos \mathrm{A} \cdot \alpha^{2}+\mathrm{G} \cos \mathrm{B} \cdot \beta^{2}+\mathrm{H} \cos \mathrm{C} \cdot \gamma^{2}\right.} \\
-(\mathrm{F}+\mathrm{G} \cos \mathrm{C}+\mathrm{H} \cos \mathrm{B}) \beta \gamma-(\mathrm{G}+\mathrm{H} \cos \mathrm{A}+\mathrm{F} \cos \mathrm{C}) \gamma a \\
-(\mathrm{H}+\mathrm{F} \cos \mathrm{B}+\mathrm{G} \cos \mathrm{A}) \alpha \beta] \\
-\lambda^{2}\left[\mathrm{~F}^{2} \alpha^{2}+\mathrm{G}^{2} \beta^{2}+\mathrm{H}^{2} \gamma^{2}-2 \mathrm{FG} \alpha \beta-2 \mathrm{GH} \beta \gamma-2 \mathrm{HF} \gamma \alpha\right]=0 .
\end{gathered}
$$

We may now determine the circumscribed conics that are confocal with inscribed conics, that is, that have isogonal points for foci. In the tangential equation, the result of putting one of the coordinates equal to zero must be a complete square; or, in the point-equation, the coefficients of $\alpha^{2}, \beta^{2}, \gamma^{2}$ must vanish. Thus we obtain the conditions

$$
\lambda F= \pm 1+\cos A, \lambda G= \pm 1+\cos B, \lambda H= \pm 1+\cos C ;
$$

and the equations of the circumscribed conics are (in tangential coordinates)

$$
\alpha^{2}+\beta^{2}+\gamma^{2} \pm 2 \alpha \beta \pm 2 \beta \gamma \pm 2 \gamma \alpha=0 .
$$

Four of these eight conics degenerate into a point (the centre of the inscribed circle, or of one of the escribed circles) taken twice over. The corresponding inscribed conic, however, does not degenerate, but is the inscribed circle or one of the escribed circles. If, where the double signs occur in this equation, the three negative signs be 
taken, or two positive and one negative sign, we obtain the tangential equations of the four non-degenerate circumscribed conics that are confocal with inscribed conics. In point-coordinates, these equations become

$$
\beta \gamma \pm \gamma \alpha \pm \alpha \beta=0 \text {. }
$$

One of these conics is an ellipse and the other three are hyperbolas.

The equations of the confocal inscribed conics are, in tangential coordinates,

$$
( \pm 1-\cos A) \beta \gamma+( \pm 1-\cos B) \gamma \alpha+( \pm 1-\cos C) a \beta=0 ;
$$

and in point-coordinates

$$
[( \pm 1-\cos \mathrm{A}) \alpha]^{1 / 2}+[( \pm 1-\cos \mathrm{B}) \beta]^{1 / 2}+[( \pm 1-\cos \mathrm{C}) \gamma]^{1 / 2}=0
$$

where either the three plus signs, or two minus signs and one plus sign, are to be taken.

The centres of these conics are

$\left(1 / r_{1}, 1 / r_{2}, 1 / r_{3}\right),\left(1 / r, 1 / r_{3}, 1 / r_{2}\right),\left(1 / r_{3}, 1 / r, 1 / r_{1}\right),\left(1 / r_{2}, 1 / r_{1}, 1 / r\right)$.

The existence of these four pairs of confocal conics has been remarked by M. Émile Lemoine, ${ }^{*}$ who has shown that the normals to the circumconics at the vertices, are the internal and the external bisectors of the angles of the triangle; that the points of contact of the inscribed conics with the sides are the points of contact of the inscribed and escribed circles; and that the centres of the conics are the "centres of anti-parallel medians" of the four triangles that have for vertices the three ex-centres or the incentre and two ex-centres.

* Sur les quatre groupes de deux points d'un triangle ABC qui sont en même temps les foyers d'une conique inscrite et d'une conique circonscrite à ce triangle. Association française pour l'avancement des sciences naturelles. (Rouen.) 1883. 